\section{Nuclear Medicine Instrumentation}

\section{J. Prekeges}

Burlington, Massachusetts: Jones and Bartlett, 2013, 376 pages, $\$ 105.95$

This soft-cover textbook on nuclear instrumentation was written for an audience of nuclear medicine technology students, medical imaging technologists, nuclear medicine physicians, radiologists, medical imaging residents, biomedical engineers, and any individual interested in gaining an understanding of the topic. According to the author, the purpose of this book is to address a significant deficit of available resources for those seeking a dedicated textbook on the subject matter. This textbook is in its second edition and is presented as 19 chapters and 6 supporting appendices. The chapters are divided into 4 distinct parts that help the reader understand small instruments, $\gamma$-cameras, SPECT, and PET. The chapter on PET includes a discussion on CT and an introduction to MR imaging.

The content of the book begins with introductions to gas-filled detectors, scintillation detectors, semiconductors, and factors relating to radiation measurement. The second part addresses the specifics of $\gamma$-cameras, including image digitization and display, collimators, image characteristics, performance measures in planar imaging, and quality assurance and quality control of $\gamma$-cameras. The third part covers the details of SPECT, including image characteristics, the effect of acquisition parameters on SPECT imaging, how to improve SPECT images, and quality control and artifacts in SPECT. The fourth part focuses on PET, including instrumentation, image characteristics, performance measures and quantification, and quality control and artifacts, and concludes with CT and MR imaging. The appendices include atomic structure and interactions of high-energy radiation, basic electronics and devices, film and film processing, computer fundamentals, collimator mathematics, and laboratory accreditation. The author writes in a clear and concise fashion and assumes the reader has little or no background on nuclear medicine instrumentation. The textbook has sample calculations, tables, charts, images, and diagrams throughout, which complement the text and enable the reader to have a more comprehensive understanding of the written material. I applaud the author for including information on semiconductors, CT, and MR imaging. Unfortunately, there are limitations on the CT and MR imaging content due to the book's focus on nuclear medicine instrumentation. The CT and MR imaging sections are more or less an introduction to these imaging modalities. I would encourage readers seeking comprehensive information on CT and MR imaging to search for other dedicated instrumentation textbooks on these subjects.

I would highly recommend this textbook for anyone who is interested in teaching or learning more about nuclear medicine instrumentation. It is a welcomed addition for any medical imaging library. Readers will find it interesting and worthwhile. I have used this book since the release of the first edition in 2011 for my course in nuclear medicine instrumentation. It has replaced 3 textbooks and many handouts that I once used to teach the class. Students are always tough critics concerning textbooks, but this book has received nothing but positive comments from my students. I also provide instruction to radiology residents, and they find the book useful as well. The author has successfully written and updated a textbook that will become a mainstay in nuclear medicine-related educational programs. This textbook clearly demonstrates why experienced nuclear medicine technology program directors should consider taking the time to enhance the profession by authoring textbooks that will benefit everyone.

William L. Hubble, MA, CNMT, RT(R)(N)(C), FSNMMI-TS Saint Louis University

3437 Caroline St.

St. Louis, MO 63104

E-mail: hubblewl@slu.edu

Published online Mar. 20, 2013.

DOI: 10.2967/jnmt.113.120790 


\section{Question 1}

\section{Answers to the Questions on Page 115}

\section{Answer: B}

${ }^{67} \mathrm{Ga}$ is a ferric ion analog that binds to transferrin, which is an acute-phase reactant-a protein whose plasma concentrations increase in response to inflammation. The cells at the site of inflammation will locally express different interleukins and tumor necrosis factor- $\alpha$, which causes these serum proteins to accumulate. As a result, ${ }^{67} \mathrm{Ga}$ bound to transferrin will flow to the site of inflammation. Since it has a stronger affinity for lactoferrin, it will bind to the lactoferrin being released by neutrophils at the site of inflammation. Additionally, ${ }^{67} \mathrm{Ga}$ will bind to bacterial siderophores and neutrophil cell membranes (whether living or dead), making it a sensitive agent for chronic infections.

\section{Question 2}

Answer: A

Although the liver will appear to have the most uptake on the images, the large bowel will actually receive the highest dose of radioactivity because it is the principal method of excretion and has a slow transit time. The dose to the liver is $0.023 \mathrm{~Gy}(2.3 \mathrm{rad}) / 185 \mathrm{MBq}(5 \mathrm{mCi})$. The salivary glands will have normal physiologic uptake initially, which washes out over the next $48 \mathrm{~h}$. The small bowel typically does not accumulate ${ }^{67} \mathrm{Ga}$.

\section{Question 3}

\section{Answer: D}

Recent gadolinium administration has been observed to cause decreased ${ }^{67}$ Ga-citrate localization because it increases the level of serum ferric ions, which will saturate the receptor sites. Similarly, recent blood transfusions will cause an increased serum iron level that results in competitive inhibition of the transferrin binding sites. Poor bowel preparation or constipation will cause physiologic radiotracer accumulation, which can obscure pathologic findings. Some have advocated for laxative use before imaging with ${ }^{67} \mathrm{Ga}$; however, one risks causing inflammation that would bring about aberrant uptake. Antibiotics have not been known to cause decreased ${ }^{67} \mathrm{Ga}$ uptake, likely because they do not share any similar pharmacokinetics. In fact, one of the indications for gallium scintigraphy is to monitor antibiotic effectiveness in combating chronic infections.

\section{Question 4}

Answer: B

${ }^{67} \mathrm{Ga}$ is not as useful for inflammatory bowel disease or for abdominal or pelvic infections because abnormal accumulation is nearly impossible to distinguish from physiologic excretion. ${ }^{99 \mathrm{~m}} \mathrm{Tc}$ - or ${ }^{111} \mathrm{In}$-labeled white blood cells would be the more appropriate choice in inflammatory bowel disease. ${ }^{67} \mathrm{Ga}$ is excellent in fever of unknown origin because the long half-life allows for a greater imaging duration. It also has the advantage over ${ }^{111}$ In of localizing to sources of chronic infections or tumors, which are often incidentally discovered as the cause for fever of unknown origin. ${ }^{67} \mathrm{Ga}$ has the characteristic $\lambda$-panda sign (configuration of uptake in mediastinum and salivary glands, respectively) that is sensitive and specific for sarcoidosis. ${ }^{67} \mathrm{Ga}$ was initially used as an imaging agent for active lymphoma before largely being replaced by ${ }^{18} \mathrm{~F}-\mathrm{FDG}$ PET.

\section{Question 1}

\section{Answers to the Questions on Pages 117 and 118}

\section{Answer $=$ A}

${ }^{111}$ In-octreotide binds with high affinity to somatostatin receptor subtypes 2 and 5 .

\section{Question 2}

\section{Answer $=$ D}

Diffuse mild uptake in the lungs in this patient was within the centrilobular emphysema. One possible explanation is activation of the cells expressing somatostatin receptors, likely secondary to mild chronic inflammation associated with emphysema. 